\title{
Germanica
}

\section{Arthur Schnitzler et la Révolution. Le Perroquet vert : prologue en forme de grotesque}

Arthur Schnitzler und die Französische Revolution. Der grüne Kakadu: eine Groteske als Prolog

\section{Geneviève Roussel}

\section{OpenEdition}

Journals

Édition électronique

URL : http://journals.openedition.org/germanica/2528

DOI : $10.4000 /$ germanica. 2528

ISSN : 2107-0784

\section{Éditeur}

Université de Lille

\section{Édition imprimée}

Date de publication : 31 décembre 1989

Pagination : 71-86

ISSN : 0984-2632

\section{Référence électronique}

Geneviève Roussel, «Arthur Schnitzler et la Révolution. Le Perroquet vert : prologue en forme de grotesque », Germanica [En ligne], 6 | 1989, mis en ligne le 02 décembre 2014, consulté le 06 octobre 2020. URL : http://journals.openedition.org/germanica/2528; DOI : https://doi.org/10.4000/ germanica. 2528

Ce document a été généré automatiquement le 6 octobre 2020.

(c) Tous droits réservés 


\title{
Arthur Schnitzler et la Révolution. Le Perroquet vert : prologue en forme de grotesque
}

\author{
Arthur Schnitzler und die Französische Revolution. Der grüne Kakadu: eine \\ Groteske als Prolog
}

Geneviève Roussel

"À Vienne, toujours pas - hélas - de révolution!"

(A.S. 1899)

1 Vienne - 1899. Le conseiller aulique de la censure, alerté par une archiduchesse, procède à un réexamen de la petite comédie de Schnitzler jouée au très officiel Burgtheater depuis quelque temps. Ne serait-elle pas, sous couvert de l'Histoire, - Le Perroquet vert ${ }^{1}$ se passe à Paris le soir du 14 juillet 1789 - désobligeante pour le pouvoir viennois, voire subversive? Schnitzler fut prié de supprimer le personnage du commissaire, unique représentant, un peu benêt, de l'administration royale; de remplacer l'illustre nom de son duc de Chartres par un nom de fiction ${ }^{2}$, et de rogner le nombre des cris de Liberté, Vive la liberté. Schnitzler attribua au duc un nom digne de la comtesse de Ségur, raya quelques appels à la liberté, mais maintint son commissaire! La pièce eut encore quelques représentations, qui s'espacèrent bientôt. La censure, sans recourir à la force, savait s'y prendre.

2 Quelques semaines plus tard, même scénario à Berlin, où on désirait une version « édulcorée » de la pièce; Schnitzler fit alors remarquer à son correspondant berlinois ${ }^{3}$ : malgré les représentations du Perroquet vert, «à Vienne, toujours pas - hélas - de révolution!».

3 Les œuvres de Schnitzler furent longtemps assimilées à une littérature viennoise fin de siècle de comédies de mœurs, frivole, sceptique tout au plus. Plus récemment la critique a découvert le peintre impitoyable d'une société viennoise politiquement et moralement hypocrite, vouée à l'anéantissement (dès lors surgit le reproche fait à 
Schnitzler de n'être qu'un " poète froid, sans emportement ", « ne pouvant ni absoudre, ni condamner $»^{4}$ ).

Le Perroquet vert - une des rares pièces dites « historicisantes » de Schnitzler - serait donc une image d'Épinal surannée, pochade souriante en "nette rupture avec la politique $»^{5} .$. ou bien "l'analyse d'une révolution» et partant analyse d'un état prérévolutionnaire viennois: "l'Histoire est la métaphore d'un état de faits contemporain », «l'Histoire n'est pas là aux fins de comparaison, mais en lieu et place du présent $»^{6}$ "les protagonistes ne doivent rien au Paris révolutionnaire de 1789, ce sont des Viennois de $1900 »^{7}$ !

Renvoyant dos à dos ces lectures a priori, il convient de montrer, à partir de cette pièce exempte de toute " viennoiserie ", combien, rejetant pièces historiques, pièces à thèse, théâtres psychologique et réaliste, Schnitzler, revendiquant avant tout l'autonomie de la fiction, est ici créateur d'une forme propre à son propos. Réactualisant certains éléments dramaturgiques traditionnels (on trouverait leurs traces dans l'histoire du théâtre viennois), il organise sa pièce autour du thème de l'interaction de la fiction et de l'action, à la faveur du "jeu dans le jeu», subtile variation théâtrale de son interrogation permanente sur jeu et réalité, masque et personne. Mais il inclut en outre dans sa dramaturgie le regard du spectateur historiquement informé, pour qui les destins individuels, qui se nouent et se dénouent sur la scène au gré du dramaturge, dépendent par ailleurs d'un tout autre démiurge, extérieur au spectacle. Réfèrent implicite, la Révolution de 1789 (ou plus exactement ses versions-reflets qui vivent dans les imaginaires ... de Schnitzler, des spectateurs, des lecteurs) investit la structure théâtrale. Les catégories élémentaires du tragique et du comique n'étant pas appropriées, Schnitzler choisit celle de la tragi-comédie, et plus précisément celle du grotesque. Le Perroquet vert est donc ${ }^{8}$ " une pièce grotesque en un acte ", située "à Paris, le soir du 14 juillet 1789, dans la gargote de Prospère ».

"Cet étrange cabaret"

6 L'idée de la pièce, affirme Schnitzler, lui serait venue à la lecture d'un journal relatant la fortune d'un café-théâtre - de Londres ou de Paris - où d'authentiques acteurs jouaient à être voleurs ou assassins ${ }^{9}$. Dans son étude De la physiologie de la création c'est précisément Le Perroquet vert que Schnitzler choisit comme exemple type de l'œuvre née de l'inspiration (Einfall). « Dans un cabaret des comédiens jouent aux criminels. Et si l'un de ces comédiens était réellement criminel ?» Bien entendu, ajoute Schnitzler, la création relève rarement d'un unique élément, « dans la mesure où tout de suite après l'idée survient un second élément qui vient en aide au projet. Puis souvent un troisième et un quatrième $»^{10}$.

7 Et si le crime avait lieu sur scène ? Et dans le Paris de 1789 ?... Un bien «étrange cabaret », de fait, que ce Perroquet vert. Prospère, le patron, ancien directeur de théâtre ruiné, a engagé certains acteurs de son ancienne troupe pour y jouer, tous les soirs, en guise de spectacle, un monde interlope. Le public, noble et snob, accourt, se plaît à frissonner, participe, relance l'action, et, sous couvert de théâtre, se laisse injurier et menacer.

8 La soirée mise en scène par Schnitzler est une soirée de crise, décisive dans la vie du caveau, dont l'existence même vacille. Le pouvoir, en la personne du commissaire du quartier, enquête sur la nature subversive de ce qui s'y passe et parle d'interdiction. 
Soirée exceptionnelle du côté des spectateurs : la marquise de Lansac, flanquée de son mari et de son amant, célèbre poète officiel, décide, à la recherche de sensations toujours plus corsées, de s'encanailler en ce lieu, pour la première fois. Albin, comte de Nogeant, dix-sept ans, huron de province, découvre la vie parisienne en raccourci : les fastes traditionnels de la Cour et de l'Église le matin à Notre-Dame, les persiflages déroutants du café-théâtre le soir.

Soirée décisive pour la troupe : l'acteur spécialiste du rôle de pickpocket a été jeté en prison pour... vol à la tire. Un authentique meurtrier par contre, est embauché, au sortir de la prison, pour jouer un rôle... de meurtrier. Mais surtout, Henri, l'acteur phare de la troupe, vient d'épouser Léocadie, actrice de renom d'un théatre voisin, et promet une dernière prestation exceptionnelle, en guise d'adieu au théâtre et à Paris : il a décidé de vivre désormais une rousseauiste idylle loin de la ville. Prospère, soucieux de l'avenir de ses affaires, cherche à le retenir, avec la complicité muette de Léocadie, peu désireuse au fond d'abandonner sa vie d'aventures théâtrales et demi-mondaines. En cette soirée de crise la frontière entre jeu et réalité est plus floue que jamais, plus excitante aussi, dans ce lieu clos de l'équivoque. N'y rencontre-t-on pas des gens « qui jouent les criminels - et d'autres, qui le sont sans le savoir »?

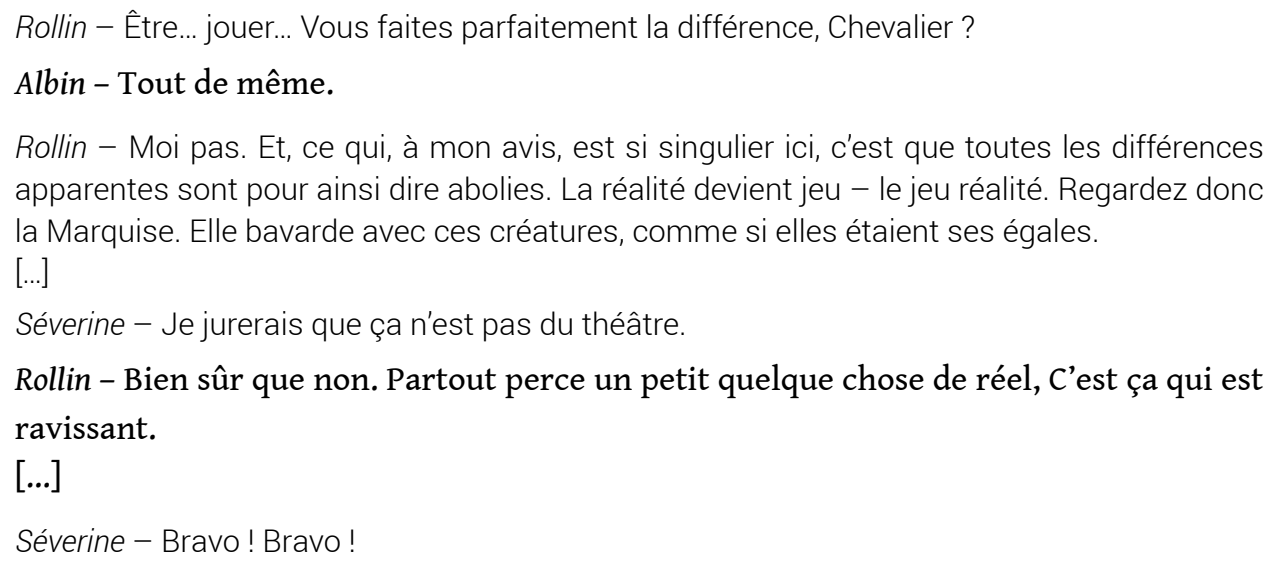

Le jeu, miroir ambigu du réel, peut devenir substitut de réalité, le théâtre exorcisme. Pour sa dernière prestation, Henri feint d'avoir surpris sa femme avec un amant, le duc de Cadignan, et raconte comment il l'a tué. Récit si haletant de vérité que tous, y compris les acteurs, croient au meurtre passionnel, d'autant plus que beaucoup savent que Cadignan est effectivement l'amant de Léocadie. La fiction - ici exorcisme inconscient: Henri ignore l'aventure de sa femme - rencontre la réalité et contraint son créateur à passer à l'acte lorsque parait Cadignan : le meurtre fictif se fait meurtre réel, qui clôt la représentation théâtrale, l'annule et la sublime.

Prospère avait promis au commissaire une représentation " anodine ", non subversive ! Elle débouche sur le meurtre d'un grand du royaume! Constatant que "tout cela dépasse les bornes ", le commissaire décide d'arrêter le meurtrier " au nom de la loi ». Mais en ce lieu, en ce jour, où sont les bornes, où est la loi ?

"Un humour inquiétant » 
La crise du Perroquet vert et la cruelle démonstration du pouvoir d'une fiction ludique sont englobées dans une plus vaste crise - une plus vaste démonstration peut-être. Le microcosme de la cave est sécrétion et emblème du monde extérieur.

$\mathrm{Au}$ perroquet vert comme dans Paris on ruse contre l'ordre établi par le désordre organisé : le mouvement des forces déstabilisatrices semble toléré, contourné, à travers tout un réseau de rites de défoulement, dérision, exorcisme.

Le duc - Ces gens se mettent à avoir un humour inquiétant.

François - Pourquoi inquiétant? Moi, ça me rassure.

Tant que ces gueux auront le goût de plaisanter, il ne se passera rien de grave.

$[\ldots]$

Le duc (à propos de Prospère qui vient de l'insulter) - Si j'était le Roi, j'en ferais mon fou du roi, ou plutôt, j'aurais beaucoup de fous du roi, lui entre autres.

[...]

François - Demain, nous irons au Palais-Royal : tu verras les discours, les blasphèmes que les gens débitent. Mais nous les laissons discourir. C'est la meilleure solution. Au fond, ce sont de braves gens, il faut les laisser se défouler comme ça.

$[\ldots]$

Prospère - Pouvoir lancer à la tête de ces gens ce que je pense d'eux et les injurier tout mon content, alors qu'ils croient que je plaisante - ça suffit à mon bonheur. Ça aussi c'est un moyen de passer sa fureur.

[...]

François - Ce ne sont que des braillards, des bavards, des guignols... Aujourd'hui, pour changer, ils hurlent devant la Bastille comme ils l'ont déjà fait cinq six fois !

Défoulement snob dans la cave, défoulement populaire dans les rues de Paris : le dixhuitième siècle est à l'œuvre, propos libertins et caustiques ici, là discours séditieux, simples discours!

Grasset - Ils sont en route vers la Bastille... et je peux le dire : ils ont suivi mon appel. Je te le jure, nous allons la prendre, avant ce soir.

Prospère - Oui, bien sûr... si vos discours pouvaient faire tomber les murailles !

17 Grasset, l'ex-acteur devenu émule de Desmoulins, a beau se montrer sûr de lui, confiant dans le pouvoir des idées, proclamer que le temps n'est plus aux brochures ni aux discours, mais qu'est venu le moment de passer aux actes, pour les protagonistes du Perroquet vert ce 14 juillet semble devoir faire date dans leur calendrier personnel tout au plus.

Mais Schnitzler a pris soin d'enclore la soirée théâtrale du cabaret dans le laps de temps de la prise de la Bastille (ramené à quelques heures pour les besoins du dramaturge). Au propos téméraire de Grasset dans la première scène - «nous allons la prendre, avant ce soir »-, répond l'écho de la dernière scène : « elle est à nous, elle est à nous. Elle est tombée, la Bastille est tombée ».

19 Aux acteurs et au public du Perroquet vert, restés sourds aux bruits de tumulte, cette Bastille prise permet la pirouette finale: le meurtre passionnel perpétré sur la personne d'un noble est élevé au rang de haut-fait au service du peuple : «l'assassin d'un duc est un ami du peuple ».

Le spectateur a, quant à lui, tout au long de la pièce, tendu l'oreille... 
Dehors, sans que les hôtres du caveau y prennent garde, le tonnerre de l'histoire s'est mis à rouler plus vite.

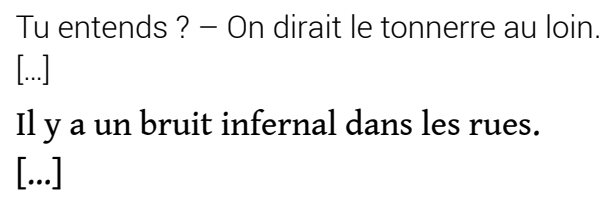

Schnitzler reprend ici le leitmotiv sonore et littéraire des premières pages des Origines de la France contemporaine de Taine ${ }^{11}$, à qui il emprunte par ailleurs quelques anecdotes concrètes, telle celle du maire de Lelange devant qui le peuple fait défiler un cercueil vide - ici encore jeu plus menaçant qu'innocent.

Schnitzler, bien sûr, fait œuvre de fiction. Mais seules une lecture superficielle de la pièce, une mauvaise connaissance du dix-huitième siècle français ont pu amener les commentateurs étrangers à voir dans la pièce un non-sens historique, dans le cadre historique français un masque plaqué sur le visage viennois des années 1900. Schnitzler, grand amateur, surtout dans la première partie de sa vie, de théâtre et de littérature français, propose, avec son caveau du Perroquet vert, où se côtoient nuitamment nobles et comédiens, filles et marquises, poètes et filous, et où la satire le dispute à la licence, non quelque café littéraire viennois, mais un lieu de rendez-vous tout droit issu de Restif de la Bretonne ${ }^{12}$ et de ses Nuits de Paris. Les protagonistes ne sont pas les grands bourgeois héritiers fragiles d'une fin de siècle esthète et libérale. Marginaux, certes, mais avant tout bien de leur époque. Les nobles n'oublient pas leurs réflexes de caste, même s'ils s'encanaillent. Et les comédiens pensent d'abord et surtout à gagner leur vie - en s'offrant en outre, chez Prospère, le plaisir de l'improvisation et le luxe de la raillerie. Tous sont jeunes, très jeunes - ils ont ce dynamisme et cette jeunesse qui frappe tant les historiens de la Révolution - : de dix-sept à vingt-quatre ans pour les habitués de la taverne, les comédiens et leurs comparses, quelques années de plus pour le marquis de Lansac; le commissaire lui-même, une fois débarrassé de son uniforme, est un "élégant jeune homme "! Leur époque, "une époque agitée ", comme le dit le commissaire, est celle de Rousseau, du rêve que fait Henri de «fuir. Vers la solitude, la campagne, la grande paix [...] et un calme infini, le ciel merveilleux et consolateur au-dessus de nous». Et celle de Restif et de ses nuits libertines. Celle aussi de Cerrutti, de Desmoulins et de ses discours du Palais-Royal. «D'où viens-tu, Grasset, du Palais-Royal ? [...] Grasset - Sais-tu après qui j'ai parlé ? Après Camille Desmoulins! » Si l'on agite des idées utopiques ou séditieuses, si, en province, il y a des troubles plus sérieux - on a tué le maire de Toulon, pillé Brignolles - c'est que l'état de la nation n'est guère brillant : «À la campagne, on meurt de faim. Les gens sont encore mille fois plus malheureux qu'en ville [...]. Bientôt il n'y aura plus de blé nulle part en France. La misère à coup sûr ", prédit Prospère. "Les paysans se mettent à être impertinents... On ne sait plus que faire ", remarque un membre de la noblesse. "Que veux-tu?, les pauvres diables ont faim, c'est ça le fin mot de l'affaire ", répond l'un de ses pairs. Toute cette agitation n'est que provisoire, exutoire? Certes. Le duc pourtant laisse entendre : «Si j'étais le Roi, j'aurais mis fin à tout cela... depuis longtemps ».

Schnitzler n'évacue donc pas la réalité historique. Pas plus que les rapports sociaux. Ils sont partout patents, au cœur même de l'ambiguïté des identités déguisées ; Schnitzler, ni idéologue, ni prophète, montre l'ennui insouciant de jeunes nobles installés dans 
leurs privilèges, les besoins, la hargne d'autres représentants de la société. Dans la stricte immanence de sa pièce, il a le regard que devrait avoir, selon lui, l'historien, «celui qui est capable de vivre en esprit le passé comme du présent: c'est-à-dire de ressentir, alors qu'il s'occupe du déroulement d'un événement qu'il connaît parfaitement, son issue comme encore incertaine ${ }^{13}$.

Le 14 juillet 1789 de Schnitzler est conforme à celui de l'Histoire, il est encore le jour de tous les possibles.

$$
\begin{array}{r}
\text { "La tragédie moderne se mue - inéluctablement - } \\
\text { en tragi-comédie " }
\end{array}
$$

Sous le titre de presque toutes ses œuvres théâtrales Schnitzler note simplement " pièce " (Schauspiel) en un, ou en trois, cinq actes. Il ne précise que dans des cas très particuliers, par exemple " pantomime » ou « burlesque ». Sous le titre du Perroquet vert figure la mention " pièce en un acte $»^{14}$. Il s'agit donc d'une forme théâtrale bien précise dans l'esprit de l'auteur ${ }^{15}$. Les commentateurs pour leur part mentionnent le terme avec une attention distraite, lui attribuant approximativement le sens de «caricatural »; les études sur le grotesque ignorent souvent la pièce, où l'écartent de manière plus ou moins abrupte : Kayser, par exemple, affirme : «il fallait bien l'étudier, malgré sa faible rentabilité pour notre sujet ", mais " elle n'a pas grand-chose à voir avec notre conception du grotesque $»^{16}$ ! La pièce de Schnitzler mériterait pourtant d'être étudiée comme archétype de certaines formes du théâtre grotesque du vingtième siècle.

Pourquoi Schnitzler choisit-il, à l'aube de ce vingtième siècle, pour évoquer un des tout derniers instants d'équilibre précaire d'un monde tout frémissant déjà de séismes imminents, la forme du grotesque? Ce recours ne signifie ni vision grotesque du monde, ni démonstration aux fins de quelque apologie. Le grotesque est ici une structure esthétique cohérente, sans doute une des mieux adaptées à la psyché moderne, puisque, Schnitzler le souligna bien des années avant Dürrenmatt, «la tragédie moderne se mue - inéluctablement - en tragi-comédie $»^{17}$.

La pièce évolue sur l'étroite ligne de partage du tragi-comique. Le prétexte du théâtre dans le théâtre permet d'écarter à la fois le tragique et la dérision, et, en soulignant le caractère interchangeable du tragique et du comique, de maintenir jusqu'au dernier instant le principe de l'ambigu. Le caveau de Prospère se veut lieu de divertissement. Le divertissement est autre chose que le jeu considéré comme passe-temps: il n'est pas naïf. Détournement du monde et de soi-même, il sied particulièrement au dix-huitième siècle. Chez Prospère les acteurs jouent à faire comme s'ils n'étaient pas des acteurs (wie wenn, als ob ponctuent la pièce, appliqués tant au réel - historique - qu'au fictif), les spectateurs jouent à faire comme s'il ne s'agissait pas de théâtre : le divertissement est dans cette convention même. En sortir c'est se priver du plaisir. Pas plus que les acteurs ne doivent donner du «directeur » à Prospère, les spectateurs ne doivent applaudir, même s'il s'agit de rompre une tension trouble ${ }^{18}$. En sortir serait laisser s'engouffrer le bien et le mal, le sens moral : pas question donc pour un acteur de jouer «l'homme aux remords ", encore moins d'évoquer, au moment où les juges parisiens pourraient finir «à la lanterne ", le juge suprême, l'instance immortelle de la conscience de chacun ${ }^{19}$. S'accrocher à cette convention, c'est se prémunir contre l'angoisse. François répète sans cesse à Albin, qui a du mal à démêler jeu et réalité, que tout n'est que divertissement (Spaß, alles sur Spaß!). Jusqu'au moment où Albin, provincial moins perverti par le factice que son ami François ${ }^{20}$, s'aperçoit que les insolences des acteurs, 
les prétendus mots d'esprit correspondent à de réelles menaces et crie que « ces gens pensent ce qu'ils disent !». En fait, comme le dit Scaevola, le public a, sans le savoir, la gaieté un peu forcée de gens qui seront pendus sous peu !21

Prospère qui, dans son antre, mène le jeu, appelle de ses vœux, dès le début de la pièce, le jour béni où ce qui n'est encore que divertissement deviendra réalité (aus dem Spaß wird Ernst) ${ }^{22}$. Pour le moment il se contente du plaisir (Vergnügen) de canaliser sa fureur et sa haine du public (dont il est toutefois éminemment complice, quel directeur n'aurait cette complaisance !) en insultes vigoureuses. Mais son poignard pourrait, ditil, jouer un jour un autre rôle que celui d'accessoire de théâtre émoussé. Dans la dernière scène, volant au secours de la victoire, comprenant subitement, en homme de théâtre qui a le sens du grandiose, le poids symbolique de la prise de la Bastille, il met fin à son rôle de directeur de troupe en le concluant par un double et retentissement adieu sans équivoque : fini de jouer! (Der Spaß ist zu Ende). Mais la grimaçante comédie de la troupe, le plaisir ambigu pris par le noble public, ont permis au lecteur de déceler les tensions, les fissures, et de voir spectacle, acteurs et spectateurs dans un éclairage critique, de deviner d'inquiétantes perspectives.

Ancrant sa pièce dans l'ordre du tragi-comique, Schnitzler précise ce choix en élisant la forme grotesque. Renouant avec l'origine même de la "grottesque ", cet ornement de fantaisie qui entrelace les règnes végétaux, animaux et humains, né dans les ténèbres des " grottes ", ruines romaines revisitées par la Renaissance, se muant en "singeries " satiriques et fantastiques dans le dix-huitième français ${ }^{23}$, Schnitzler donne pour cadre à sa pièce l'antre de Prospère (Spelunke, l'étymologie est parlante !), reflet en creux et en réduction du monde dressé au dehors, caverne platonicienne où les ombres chinoises évoluent dans un monde apparemment marginal, mais que sept marches seulement séparent du pavé de Paris. Ce monde clos, circulaire et sombre est celui de la permissivité, de l'identité masquée et démasquée. Il ne s'agit pas seulement de l'impertinence codifiée de la commedia dell'arte. Le grotesque va au-delà du mélange une des règles originelles de la tragi-comédie - de sujets triviaux et nobles et de la juxtaposition explosive sur une même scène des grands de ce monde et des petits, les uns singeant les autres, aux fins de divertir un public inlassablement complice. Ici, derrière les rôles dévolus par la société, le théâtre, toutes les identités sont divisées, ambivalentes, indéterminées, paradoxales; renversements et contrefaçons ne renvoient pas à un ordre provisoirement mis entre parenthèses, mais expriment intrinsèquement l'absence d'ordre, de cohérence, de frontières entre les êtres et à l'intérieur même des êtres. L'ordonnancement établi par les positions sociales, les situations, la psychologie, n'est qu'une forme plaquée, modulable à l'infini. Le grotesque, contrairement au burlesque, dénonce les distorsions internes à l'homme ; il relève du scepticisme; démystificateur, il fait éclater les conventions, tout en désamorçant le tragique. Il est par là éminemment déstabilisateur et anti-conformiste.

31 Schnitzler s'en donne à cœur-joie pour brouiller les cartes de l'ordre social, montrer son inanité, et mettre à nu des individus. Bien sûr les acteurs, adoptant pour un moment tel ou tel masque, rappellent le principe même de l'illusion théâtrale, ici plus sensible puisque font défaut les repères de la rampe, du texte de l'auteur dramatique. Mais les acteurs, masqués ou non, et leurs nobles spectateurs sont identiques, voire interchangeables. Les procédés grotesques du retournement, de la symétrie, du double, sont maniés implacablement par Schnitzler. À chaque personnage principal du groupe des acteurs fait face son double dans la catégorie des spectateurs. Ainsi le duc de 
Cadignan est-il le double de l'acteur Henri ; ils ont la même aura vibrante, qui fait d'eux le centre d'intérêt dès qu'ils paraissent. Le duc, sincère admirateur d'Henri, aimerait, s'il n'était déjà doté de la fonction de duc, être lui-même un acteur, cet être qui a le merveilleux pouvoir d'être multiple de façon crédible; «si je n'étais pas le duc de Cadignan, j'aimerais être un comédien comme lui ». Il n'hésite d'ailleurs pas à laminer en paroles - les repères sociaux, affirmant par exemple que Léocadie est née pour être "la plus merveilleuse fille de joie du monde ", car "il faut être doué pour être fille de joie - comme pour être conquérant ou poète ». Léocadie, la frivole actrice qui vient d'épouser Henri sans renoncer à sa vie libertine, n'a pourtant pas la rouerie perverse de la marquise de Lansac, son double bien né, qui, sous les yeux de mari et amant, joue les jolis cœurs avec tout frais minois masculin et ressent les troubles de la rue, la mort même du duc, comme d'émoustillants aphrodisiaques ${ }^{24}$. Le talent ne distingue pas de classe, pas plus que la luxure. Le poète officiel Rollin, amant de la Marquise, adopte prudemment le ton, les manières du monde huppé où il évolue. Son double populaire, le soi-disant philosophe Grasset, émule de fraîche date de Desmoulins, révolutionnaire par opportunisme, fait preuve d'un fanatisme prosélyte envahissant, tout en songeant à se recaser dans le théâtre s'il « ratait (sa) carrière politique ", et reconnaît que c'est en déclamant chez Prospère des discours révolutionnaires pastiches qu'il s'est mis à « haïr ces chiens en beaux habits, parfumés et pourris »!

Acteurs et sosies, doubles et couples, jouant à se singer les uns les autres, présentent de troublantes similitudes. La mouvance, le paradoxe sont à l'intérieur des êtres; en cette soirée historique, c'est aux leviers intimes que Schnitzler attribue le plus grand pouvoir de déstabilisation, de déstructuration : Éros, Thanatos, ambition, passion ou rêve. Dans une spirale toujours plus rapide on voit l'acteur devenir révolutionnaire par ambition, les nobles chercher de nouveaux frissons auprès d'une fausse plèbe, le rousseauiste pacifiste rêver de meurtre, et passer à l'acte ; la tête de de Launay se promène sur une pique, la Bastille tombe, le cadavre du duc n'est plus celui d'un rival amoureux, mais celui d'un ennemi de la liberté : « nulle part le cri de Vive la liberté ne sonne mieux que devant le cadavre d'un duc ! ». Le commissaire se ridiculise en faisant appel à l'ordre, la mesure, la loi. "Les lois, c'est nous qui les faisons», proclamme Grasset. Passant instantanément, lui aussi, au registre collectif, Prospère renchérit: «Paris nous appartient !».

Les forces centrifuges, à l'œuvre dès le départ, font voler en éclat l'univers du Perroquet vert, où chacun pouvait encore se consacrer à lui-même, à l'art, au divertissement. Happés par le tourbillon du collectif, les destins singuliers fondent, et se fondent dans le devenir de la masse. Au sortir du caveau les individus vont être, volens nolens, asservis aux sommations de l'Histoire.

"Alors que et tragique et comique sont circonscrits dans le fini, le grotesque ouvre sur l'infini "

Le Perroquet vert confirme, semble-t-il, le parallélisme entre les points saillants de l'histoire du grotesque et les périodes de crise, de rupture. La Révolution française futelle cette "immense tragi-comédie de l'humanité ", «la plus terrible grotesque de l'époque $~^{25}$, dont parle Schlegel ? Dans la pièce de Schnitzler la Révolution n'est pas nommée : un Martien ignorant tout de l'histoire de notre planète pourrait l'apprécier au premier degré, sans la référence historique. Et pourtant la Révolution est bien le pivot même de la pièce, mais pivot extérieur, décentré, qui fonde le recours à la 
structure grotesque, celle-ci désignant in fine quelque chose qui ne peut plus être représenté, appréhendé dans sa globalité. Si - le grotesque servant de révélateur - il y a diagnostic historique, il sera oblique et réservé au lecteur, au spectateur, en fonction de leur propre point de vue et de leur sensibilité.

Dans quelques brèves notes - largement postérieures au Perroquet vert - sur des sujets historiques, politiques, Schnitzler se montre sceptique quant aux devenirs des révolutions, qu'il convient de ne pas mythifier à la légère. "La nature même des révolutions veut », écrit-il, "que les pédants ne les comprennent pas, les gredins les détournent, et la masse les subisse comme une fatalité $»^{26}$. La Révolution française toutefois reste chère à son cœur ; elle est exceptionnelle et définitivement positive, reconnaît-il, car « elle commença tout de même comme une révolution de l'esprit. Il s'agissait d'obtenir la liberté, la liberté de l'esprit, et cette idée originelle continua à être opérante, même lorsque les Jacobins se déchaînèrent $"^{27}$.

Dans sa pièce Schnitzler se situe dans le domaine de la fiction: l'esthétique a charge d'ultime conviction. Le grotesque est ici retenu comme structure infiniment signifiante, puisque «alors que et tragique et comique sont circonscrits dans le fini, le grotesque ouvre sur l'infini $»^{28}$.

Dans le théâtre grec, précédant l'action et le premier chant du chœur, un prologue préparait le spectateur. Le prologue, selon Littré, est un « ouvrage qui sert de prélude à une pièce dramatique ", le prélude lui-même étant à l'origine une forme musicale non mesurée, chargée d'introduire des formes faisant appel au rythme, à la mesure du temps. En 1789 l'Histoire s'est chargée d'écrire la pièce dramatique, Schnitzler, avec Le Perroquet vert, en a écrit un possible prologue, en forme de grotesque.

Le perroquet ne serait-il pas - au même titre que la chauvesouris et quelques autres volatiles depuis les Oiseaux d'Aristophan $\mathrm{e}^{29}$ - un oiseau typiquement grotesque, lui dont le ramage singe le langage des hommes? Quelle judicieuse enseigne pour un cabaret du Palais-Royal en 1789 ! Les rois de France, rapporte Villefosse, faisaient arrêter et juger les perroquets de Paris qui tenaient des propos séditieux! Malicieux et ambigu jusque dans le titre de sa pièce, Schnitzler attribue à son perroquet la couleur verte : vert abhorré des hommes de théâtre, parce qu'il porte malheur..., vert aussi de l'espoir, couleur des feuilles des arbres, choisie au Palais-Royal par Camille Desmoulins comme signe de ralliement, à l'aube de la Révolution.

\section{NOTES}

1. - Arthur Schnitzler, Der grüne Kakadu, dans: Arthur Schnitzler, Gesammelte Werke. Die Dramatischen Werke, 1, S. Fischer Verlag, Frankfurt am Main, 1981, p. 515-552.

Première à Vienne au Burgtheater le 1.3.1899 (la pièce sera jouée pour la dernière fois le 18 mai 1899, reprise en 1900, 1905, etc.) en même temps que Die Gefährtin et Paracelsus.

Première à Berlin au Deutsches Theater le 29.4.1899.

Le Perroquet vert fut joué à Paris en 1903 (première le 7.11) au Théâtre Antoine, avec Antoine, qui appréciait beaucoup la pièce, dans le rôle de Prospère, dans la traduction de Maurice Vaucaire 
(1866-1918).

Première publication dans Die Neue Deutsche Rundschau, 10, Heft 3 ; première édition : Der grüne Kakadu. Paracelsus. Die Gefährtin. Drei Einakter, S. Fischer, Berlin, 1899, p. 95-178.

Sur les interdictions et la censure, voir : A.S., Briefe 1875-1912, S. Fischer Verlag, Frankfurt, 1981, p. 362-368 et 848-858.

2. - Le duc de Chartres de Schnitzler était déjà de fiction! Puisqu'il ne peut historiquement correspondre ni au futur Louis Philippe, né en 1773 (16 ans en 1789, le duc de Schnitzler a 24 ans), ni à son père, Louis Philippe Joseph (1747-1793), duc de Chartres depuis 1752, cousin de Louis XVI, qui vivait au Palais-Royal, centre d'agitation révolutionnaire, et vota la mort du roi.

3. - Lettre du 10.3.99 à Ludwig Fulda, A.S., op. cit., p. 369 : « in Wien ist (leider) noch immer keine Revolution ausgebrochen ».

4. - Carl Schorske, «Politique et psyché : Schnitzler et Hofmannsthal », dans Vienne fin de siècle, Editions du Seuil, Paris, 1983, p. 30.

5. - Hartmut Scheible, Arthur Schnitzler, Rowohlt Taschenbuch Verlag, Reinbeck, 1976, p. 73.

6. - R. Urbach, op. cit., p. 43.

7. - Ursula Keller, Böser Dinge hübsche Formel. Das Wien Arthur Schnitzlers, Verlag Guttandin und Hoppe, 1984, p. 195.

8. - A.S., op. cit., p. 515. Sous-titre et indication scénique : «Groteske in einem Akt». «Spielt in Paris am Abend des 14. Juli 1789 in der Spekunke Prospères».

9. - Lettre du 1.10.1902 à Stephan Epstein, op. cit., p. 451.

10. - A.S., "Aphorismen und Betrachtungen», dans: Gesammelte Werke, op. cit., 1967, Materialien zu einer Studie über Kunst und Kritik, p. 380-381.

11. - Hippolyte Taine (1828-1893), Les Origines de la France contemporaine (1875-1894).

Schnitzler : grand lecteur de Taine (dès 92), de Restif, mais aussi, p. ex. de V. Sardou, et, bien sûr, d'Alexandre Dumas !

12. - Restif de la Bretonne (1734-1806), Les Nuits de Paris.

13. - A.S., Aphorismen, op. cit., Der Geist im Wort und der Geist in der Tat, p. 148.

14. - Voir supra, note 8.

15. - Malgré de nombreuses approches, une analyse - et une histoire - de la pièce grotesque en tant que forme théâtrale autonome reste à faire. Le numéro d'Études germaniques, Le grotesque, 43, 1988, nºl, trace d'intéressantes perspectives.

16. - Wolfgang Kayser, Das Groteske in Malerei und Dichtung, Rowohlt, München, 1961, p. 98.

17. - «Die moderne Tragödie wird mit Notwendigkeit zur Tragikomödie», A.S., «Werk und Widerhall», dans Aphorismen, op. cit., p. 101.

On pense aux analyses de Dürrenmatt sur le tragi-comique obligé du $\mathrm{XX}^{\mathrm{e}}$ siècle et à sa célèbre formule: «unsere Welt hat ebenso zur Groteske geführt wie zur Atombombe», Friedrich Dürrenmatt, «Theaterprobleme», 1955, dans: Theater-schriften und Reden, Verlag der Arche, Zürich, 1966, p. 122.

18. - «Der ganze Spaß geht flöten, wenn du mich "Direktor" nennst».

19. - « Grain - Tod den Richtern! Jules - Ja ... es gibt doch vielleicht einen, den wir nicht vernichten können. Guillaume - Den Richter in uns. Prospère (leise) - Das ist abgeschmackt. Laß das. Scaevola! Brülle. Jetzt ist der Moment».

20. - Il continue à utiliser des notions comme wirklich, wahr, nicht wahr, et manifeste son malaise par les mots de wirr, verwirrt, jusqu'au cri final : « die Leute meinen es ernst».

21. - « Das Publikum hat die Lustigkeit von Leuten, die nächstens gehenkt werden ».

22. - Prospère dans la première scène : «Irgendeinmal kommt der Tag, wo aus dem Spaß Ernst wird ». Dans la dernière scène : « Der Spaß ist zu Ende [...]. Der Spaß ist zu Ende».

23. - Sur cette filiation voir le passionnant petit livre d'André Chastel, La grottesque, édition Le Promeneur, Paris, 1989, appliqué aux beaux-arts. Il serait peut-être bon de revenir, dans le domaine littéraire également, au terme originel de «la grottesque» pour désigner la forme 
littéraire. On peut regretter que dans une rapide allusion à Voltaire, Klee, etc., le nom de Schnitzler, cité fort à propos, soit défiguré par une coquille !

24. - « Séverine - Rollin, warten Sie heut Nacht vor meinem Fenster [...] Wir wollen eine schöne Stunde haben - ich fühle mich angenehm erregt».

25. - «Man kann sie (die Revolution) auch betrachten als die furchtbarste Groteske des Zeitalters, wo die tiefsinnigsten Vorurteile und die gewaltsamsten Ahndungen desselben in ein grauses Chaos gemischt, in einer ungeheuren Tragikomödie der Menschheit so bizarr als möglich verwebt sind », Friedrich Schlegel, Fragmente aus dem Athenäum ( $\mathrm{Nr} 424)$, dans Schriften und Fragmente, Stuttgart, Kröner-Verlag, 1956, p. 104.

26. - «Es liegt im Wesen der Revolutionen, daß sie von Pedanten mißverstanden, von Bösewichtern mißbraucht und von der Masse als Schicksal hingenommen werden ». A.S., [Politik, Gesellschaft], dans Aphorismen, op. cit., p. 240 (non daté).

27. - « Die Revolution des Proletariats konnte man letzten Endes gelten lassen ; mehr als das, sie war eine Notwendigkeit, wofür der beste Beweis ist, daß sie kam - eine Notwendigkeit, wenn auch, oder obwohl sie nicht gelang. Aber schlimmer ist, daß diese Revolution des Proletariats sich selber proletarisiert hat, in einer kläglicheren Weise als es die französische 1789 tat. Jene begann doch immerhin als eine Revolution des Geistes. Um Befreiung handelte es sich, um Befreiung des Geistes, und diese Uridee wirkte immer noch nach, selbst als die Jakobiner wüteten. [...] », Id., p. 233-234 (ces réflexions datent de 1918).

28. - «Während sowohl das Tragische, als das Komische innerhalb des Endlichen beschlossen ist, weist das Groteske ins Grenzenlose », A.S., Werk und Widerhall, ibid., p. 106.

29. - Schnitzler envisagea de traduire cette pièce d'Aristophane.

Les traductions récentes de la pièce de Schnitzler choisissent l'intitulé Au perroquet vert; le titre allemand - Der grüne Kakadu - permet un spectre beaucoup plus large d'allusions que la simple enseigne, mentionnée dans les indications scéniques, du cabaret Zum grünen Kakadu. Le "perroquet" français qui se substitue au "cacatoès" allemand est bien venu. Le Kakadu allemand évoque des comptines enfantines, des jeux du début du siècle, et - pourquoi pas toutes les plaisanteries possibles sur k. u. $k$ !

\section{RÉSUMÉS}

Avec Le Perroquet vert, pièce en un acte qu'il situe à Paris le soir de la prise de la Bastille, Schnitzler écrit un possible prologue à la Révolution. Acteurs et nobles posent, sous couvert de divertissement - exorcisme et catharsis ambiguë -, le problème de l'identité, celui de l'interaction de la fiction et de l'action, tout en étant figures d'un quatorze juillet conforme à l'Histoire. La Révolution sert de réfèrent implicite. Pivot extérieur à la pièce, elle fonde le recours au grotesque, analysé ici comme structure esthétique signifiante.

Mit dem Einakter Der grüne Kakadu, der am Abend der Erstürmung der Bastille in Paris spielt, schreibt Schnitzler einen möglichen Prolog zur Französischen Revolution. Schauspieler und Adlige werfen unter dem Deckmantel des Spaßes - Exorzismus und zweideutige Katharsis - das Problem der Identität, der Wechselwirkung von Geist und Tat auf, während sie zugleich Figuren eines der Geschichte entsprechenden 14. Juli sind. Die Französische Revolution dient als impliziter Bezugspunkt. Außerhalb des Stückes befindliche Achse, rechtfertigt sie doch die Verwendung des Grotesken, das hier als ästhetische, sinngebende Struktur analysiert wird. 
AUTEUR

GENEVIĖVE ROUSSEL

Université de Lille III 